\title{
On upper and lower bounds of the numerical radius and an equality condition
}

\author{
by \\ TAKEAKI YAMAZAKI (Yokohama)
}

\begin{abstract}
We give an inequality relating the operator norm of $T$ and the numerical radii of $T$ and its Aluthge transform. It is a more precise estimate of the numerical radius than Kittaneh's result [Studia Math. 158 (2003)]. Then we obtain an equivalent condition for the numerical radius to be equal to half the operator norm.
\end{abstract}

1. Introduction. For a bounded linear operator $T$ on a complex Hilbert space $\mathcal{H}$, we denote the operator norm and the numerical radius of $T$ by $\|T\|$ and $w(T)$, respectively. It is well known that $w(T)$ is an equivalent norm of $T$, since (see [5, Theorem 1.3-1])

$$
\frac{1}{2}\|T\| \leq w(T) \leq\|T\| .
$$

Concerning the second inequality, Kittaneh [8] has shown the following precise estimate of $w(T)$ by using several norm inequalities and ingenious techniques:

$$
w(T) \leq \frac{1}{2}\|T\|+\frac{1}{2}\left\|T^{2}\right\|^{1 / 2} .
$$

Obviously, (1.2) is sharper than the right inequality of (1.1). We remark that we cannot compare $w(T)$ with $\left\|T^{2}\right\|^{1 / 2}$, generally. In fact, if $T=\left(\begin{array}{ll}0 & 1 \\ 0 & 0\end{array}\right)$, then $0=\left\|T^{2}\right\|^{1 / 2}<w(T)=1 / 2$; but if

$$
T=\left(\begin{array}{lll}
0 & 1 & 0 \\
0 & 0 & 1 \\
0 & 0 & 0
\end{array}\right),
$$

then $1 / \sqrt{2}=w(T)<\left\|T^{2}\right\|^{1 / 2}=1$.

We obtain a sufficient condition for $w(T)=\frac{1}{2}\|T\|$ to hold from (1.1), (1.2) and [8]: if $T^{2}=0$, then $w(T)=\frac{1}{2}\|T\|$. But this condition is not

2000 Mathematics Subject Classification: Primary 47A12; Secondary 47A30, 47A20.

Key words and phrases: numerical radius, Aluthge transform, normaloid operators. 
necessary: if $T=1 \oplus\left(\begin{array}{ll}0 & 2 \\ 0 & 0\end{array}\right)$, then $w(T)=\frac{1}{2}\|T\|=1$, but $T^{2} \neq 0$. We remark that some (necessary or sufficient) conditions for $w(T)=\frac{1}{2}\|T\|$ to hold are given in [5, Theorems 1.3-4 and 1.3-5], but no equivalent condition has been known yet.

Let $T=U|T|$ be the polar decomposition of $T$. The Aluthge transform $\widetilde{T}$ of $T$ is defined by $\widetilde{T}=|T|^{1 / 2} U|T|^{1 / 2}$ (see [1]). The following properties of $\widetilde{T}$ are well known:

(i) $\|\widetilde{T}\| \leq\|T\|$,

(ii) $w(\widetilde{T}) \leq w(T)$,

(iii) $r(\widetilde{T})=r(T)$.

The first and last properties are easy by the definition of $\widetilde{T}$, and the second one is shown in [7], [9] and [11]. Moreover for a non-negative integer $n$, we denote the $n$th Aluthge transform by $\widetilde{T}_{n}$, i.e.,

$$
\widetilde{T}_{n}=\widetilde{\widetilde{T}}_{n-1} \text { and } \widetilde{T}_{0}=T .
$$

This was first considered in [7] and [10], independently.

In this paper, first, we obtain a more precise estimate than (1.2). In the inequality, we use a bigger term, $\|T\|$, and a smaller one, $w(\widetilde{T})$, than $w(T)$. Moreover the proof is very simple and needs only a generalized polarization identity. Next, we give a condition equivalent to $w(T)=\frac{1}{2}\|T\|$.

2. An inequality sharper than Kittaneh's. In this section, we prove a sharper estimate of $w(T)$ than Kittaneh's [8], as follows:

Theorem 2.1. For any $T \in B(\mathcal{H}), w(T) \leq \frac{1}{2}\|T\|+\frac{1}{2} w(\widetilde{T})$.

We remark that by the Heinz inequality [6], $\left\|A^{r} X B^{r}\right\| \leq\|A X B\|^{r}\|X\|^{1-r}$ for $A, B \geq 0$ and $r \in[0,1]$, we have

$$
w(\widetilde{T}) \leq\|\widetilde{T}\|=\left\||T|^{1 / 2} U|T|^{1 / 2}\right\| \leq\||T| U|T|\|^{1 / 2}\|U\|^{1 / 2}=\left\|T^{2}\right\|^{1 / 2},
$$

i.e., Theorem 2.1 is sharper than (1.2).

To prove Theorem 2.1, we use the following famous formula which is called the generalized polarization identity:

Theorem A (Generalized Polarization Identity). For each $T \in B(\mathcal{H})$ and $x, y \in \mathcal{H}$,

$$
\begin{aligned}
\langle T x, y\rangle= & \frac{1}{4}(\langle T(x+y), x+y\rangle-\langle T(x-y), x-y\rangle) \\
& +\frac{1}{4} i(\langle T(x+i y), x+i y\rangle-\langle T(x-i y), x-i y\rangle) .
\end{aligned}
$$

Proof of Theorem 2.1. First of all, we note that

$$
w(T)=\sup _{\theta \in \mathbb{R}}\left\|\operatorname{Re}\left(e^{i \theta} T\right)\right\|,
$$


since

$$
\sup _{\theta \in \mathbb{R}} \operatorname{Re}\left\{e^{i \theta}\langle T x, x\rangle\right\}=|\langle T x, x\rangle|
$$

and

$$
\sup _{\theta \in \mathbb{R}}\left\|\operatorname{Re}\left(e^{i \theta} T\right)\right\|=\sup _{\theta \in \mathbb{R}} w\left(\operatorname{Re}\left(e^{i \theta} T\right)\right)=w(T) .
$$

Let $T=U|T|$ be the polar decomposition. Then by (2.2), we have

$$
\begin{aligned}
& \left\langle e^{i \theta} T x, x\right\rangle=\left\langle e^{i \theta}|T| x, U^{*} x\right\rangle \\
& =\frac{1}{4}\left(\left\langle|T|\left(e^{i \theta}+U^{*}\right) x,\left(e^{i \theta}+U^{*}\right) x\right\rangle-\left\langle|T|\left(e^{i \theta}-U^{*}\right) x,\left(e^{i \theta}-U^{*}\right) x\right\rangle\right) \\
& \quad+\frac{1}{4} i\left(\left\langle|T|\left(e^{i \theta}+i U^{*}\right) x,\left(e^{i \theta}+i U^{*}\right) x\right\rangle-\left\langle|T|\left(e^{i \theta}-i U^{*}\right) x,\left(e^{i \theta}-i U^{*}\right) x\right\rangle\right) .
\end{aligned}
$$

Note that the inner products on the right hand side are all positive since $|T|$ is positive. Hence we have

$$
\begin{aligned}
\operatorname{Re}\left\langle e^{i \theta} T x\right. & , x\rangle \\
& =\frac{1}{4}\left(\left\langle\left(e^{-i \theta}+U\right)|T|\left(e^{i \theta}+U^{*}\right) x, x\right\rangle-\left\langle\left(e^{-i \theta}-U\right)|T|\left(e^{i \theta}-U^{*}\right) x, x\right\rangle\right) \\
& \leq \frac{1}{4}\left\langle\left(e^{-i \theta}+U\right)|T|\left(e^{i \theta}+U^{*}\right) x, x\right\rangle \\
& \leq \frac{1}{4}\left\|\left(e^{-i \theta}+U\right)|T|\left(e^{i \theta}+U^{*}\right)\right\| \\
& =\frac{1}{4}\left\||T|^{1 / 2}\left(e^{i \theta}+U^{*}\right)\left(e^{-i \theta}+U\right)|T|^{1 / 2}\right\| \quad\left(\text { since }\left\|X^{*} X\right\|=\left\|X X^{*}\right\|\right) \\
& =\frac{1}{4}\left\|2|T|+e^{i \theta} \widetilde{T}+e^{-i \theta}(\widetilde{T})^{*}\right\| \\
& =\frac{1}{2}\left\||T|+\operatorname{Re}\left(e^{i \theta} \widetilde{T}\right)\right\| \\
& \leq \frac{1}{2}\|T\|+\frac{1}{2}\left\|\operatorname{Re}\left(e^{i \theta} \widetilde{T}\right)\right\| \\
& \leq \frac{1}{2}\|T\|+\frac{1}{2} w(\widetilde{T}) \quad(\text { by }(2.3)) .
\end{aligned}
$$

Hence we have the desired inequality.

Corollary 2.2. If $\widetilde{T}=0$, then $w(T)=\frac{1}{2}\|T\|$.

Proof. The proof is easy by Theorem 2.1 and (1.1).

REMARK.

(i) In Corollary 2.2, the conditions $\widetilde{T}=0$ and $w(T)=\frac{1}{2}\|T\|$ are not equivalent: if $T=1 \oplus\left(\begin{array}{ll}0 & 2 \\ 0 & 0\end{array}\right)$, then $w(T)=\frac{1}{2}\|T\|=1$, but $\widetilde{T}=1 \oplus 0$ $\neq 0$.

(ii) The conditions $\widetilde{T}=0$ and $T^{2}=0$ are equivalent. Indeed, let $T=$ $U|T|$ be the polar decomposition. If $\widetilde{T}=0$, then

$$
T^{2}=U|T| U|T|=U|T|^{1 / 2} \widetilde{T}|T|^{1 / 2}=0 .
$$

Conversely, if $T^{2}=0$, then by (2.1) we have $\|\widetilde{T}\| \leq\left\|T^{2}\right\|^{1 / 2}=0$. 
Corollary 2.3. For $T \in B(\mathcal{H})$,

$$
w(T) \leq \sum_{n=1}^{\infty} \frac{1}{2^{n}}\left\|\widetilde{T}_{n-1}\right\| .
$$

Proof. By using Theorem 2.1 several times, we have

$$
\begin{aligned}
w(T) & \leq \frac{1}{2}\|T\|+\frac{1}{2} w(\widetilde{T}) \leq \frac{1}{2}\|T\|+\frac{1}{2}\left\{\frac{1}{2}\|\widetilde{T}\|+\frac{1}{2} w\left(\widetilde{T}_{2}\right)\right\} \\
& =\frac{1}{2}\|T\|+\frac{1}{4}\|\widetilde{T}\|+\frac{1}{4} w\left(\widetilde{T}_{2}\right) \\
& \leq \frac{1}{2}\|T\|+\frac{1}{4}\|\widetilde{T}\|+\frac{1}{8}\left\|\widetilde{T}_{2}\right\|+\frac{1}{8} w\left(\widetilde{T}_{3}\right) \leq \cdots \leq \sum_{n=1}^{\infty} \frac{1}{2^{n}}\left\|\widetilde{T}_{n-1}\right\| .
\end{aligned}
$$

Let

$$
s(T)=\sum_{n=1}^{\infty} \frac{1}{2^{n}}\left\|\widetilde{T}_{n-1}\right\| .
$$

By (2.1), $\|\widetilde{A}\| \leq\left\|A^{2}\right\|^{1 / 2} \leq\|A\|$ for any $A \in B(\mathcal{H})$, and we obtain

$$
r(T) \leq w(T) \leq \frac{1}{2}\|T\|+\frac{1}{2} w(\widetilde{T}) \leq s(T) \leq \frac{1}{2}\|T\|+\frac{1}{2}\left\|T^{2}\right\|^{1 / 2} \leq\|T\|,
$$

where $r(T)$ means the spectral radius of $T$.

It is well known that $T$ is normaloid (i.e., $\|T\|=r(T)$ ) if and only if $\|T\|=w(T)$. Here we give other conditions of normaloidity of $T$ :

Corollary 2.4. The following conditions are equivalent:

(i) $T$ is normaloid,

(ii) $\|T\|=s(T)$,

(iii) $r(T)=\frac{1}{2}\|T\|+\frac{1}{2} w(\widetilde{T})$,

(iv) $s(T)=s(\widetilde{T})$.

REMARK.

(i) In Corollary 2.4, condition (ii) cannot be replaced by the weaker condition $\|T\|=\frac{1}{2}\|T\|+\frac{1}{2}\left\|T^{2}\right\|^{1 / 2}$. For example, let

$$
T=\left(\begin{array}{lll}
0 & 1 & 0 \\
0 & 0 & 1 \\
0 & 0 & 0
\end{array}\right) .
$$

Then $\|T\|=\frac{1}{2}\|T\|+\frac{1}{2}\left\|T^{2}\right\|^{1 / 2}=1$ but $0=r(T)<\|T\|$.

(ii) In Corollary 2.4, condition (iii) cannot be replaced by the weaker condition $r(T)=w(T)$ either. In fact, let $T=1 \oplus\left(\begin{array}{ll}0 & 2 \\ 0 & 0\end{array}\right)$. Then $1=r(T)=w(T)<\|T\|=2$. (We call an operator satisfying $r(T)=$ $w(T)$ spectraloid.)

To prove Corollary 2.4, the following formula will be used.

Theorem B ([10]). For any $T \in B(\mathcal{H}), \lim _{n \rightarrow \infty}\left\|\widetilde{T}_{n}\right\|=r(T)$. 
Proof of Corollary 2.4. (i) $\Rightarrow$ (ii), (iii) and (iv) are obvious by (2.4) and $r(T)=r(\widetilde{T}) \leq s(\widetilde{T}) \leq s(T) \leq\|T\|$.

(ii) $\Rightarrow($ i). By the definition of $s(T)$,

$$
s(T)=\frac{1}{2}\|T\|+\frac{1}{2} s(\widetilde{T}) .
$$

Hence (ii) yields $s(\widetilde{T})=\|T\|$. Since $\|\widetilde{T}\| \leq\|T\|$, this gives

$$
s(\widetilde{T}) \leq\|\widetilde{T}\| \leq\|T\|=s(\widetilde{T}),
$$

and so $s(\widetilde{T})=\|\widetilde{T}\|=\|T\|$. By using the same technique, we have $\|T\|=$ $\left\|\widetilde{T}_{n}\right\|$ for all $n \in \mathbb{N}$. Hence by Theorem $\mathrm{B}$, we have

$$
\|T\|=\lim _{n \rightarrow \infty}\left\|\widetilde{T}_{n}\right\|=r(T),
$$

that is, $T$ is normaloid.

(iii) $\Rightarrow($ i). Since $r(\widetilde{T})=r(T)$, by (iii) we have

$$
r(T)=\frac{1}{2}\|T\|+\frac{1}{2} w(\widetilde{T}) \geq \frac{1}{2}\|T\|+\frac{1}{2} r(\widetilde{T})=\frac{1}{2}\|T\|+\frac{1}{2} r(T),
$$

that is, $r(T) \geq\|T\|$ and so $r(T)=\|T\|$.

(iv) $\Rightarrow$ (ii). Evident by (2.5).

In [2], Ando shows that the equality $W(T)=W(\widetilde{T})$ of numerical ranges is equivalent to $\operatorname{co} \sigma(T)=W(T)$ (i.e., $T$ is convexoid) for any matrix $T$, where $\operatorname{co} \sigma(T)$ means the convex hull of the spectrum of $T$. We think that this result is parallel to the equivalence between (i) and (iv). So we expect that $s(T)$ has some interesting properties.

3. Condition equivalent to $w(T)=\frac{1}{2}\|T\|$. In Corollary 2.2, we have obtained a sufficient condition for $w(T)=\frac{1}{2}\|T\|$ to hold. Some (necessary or sufficient) conditions for $w(T)=\frac{1}{2}\|T\|$ to hold are given in [5, Theorems 1.3-4 and 1.3-5]. But no condition equivalent to $w(T)=\frac{1}{2}\|T\|$ has been known. In this section, we give such a condition:

Theorem 3.1. Let $T \in B(\mathcal{H})$. The following conditions are equivalent:

(i) $w(T)=\frac{1}{2}\|T\|$,

(ii) $\|T\|=\left\|\operatorname{Re}\left(e^{i \theta} T\right)\right\|+\left\|\operatorname{Im}\left(e^{i \theta} T\right)\right\|$ for all $\theta \in \mathbb{R}$.

We remark that (ii) cannot be replaced by " $\|T\|=\left\|\operatorname{Re}\left(e^{i \theta} T\right)\right\|+\left\|\operatorname{Im}\left(e^{i \theta} T\right)\right\|$ for some $\theta \in \mathbb{R}$," because if $T$ is a non-zero self-adjoint operator, then $\|T\|=\|\operatorname{Re} T\|+\|\operatorname{Im} T\|=\|\operatorname{Re} T\|$, but $w(T)=\|T\|>\frac{1}{2}\|T\|$.

To prove Theorem 3.1, we need the following theorem:

Theorem $\mathrm{C}([3])$. Let $A, B \in B(\mathcal{H})$ be non-zero. Then the equality $\|A+B\|=\|A\|+\|B\|$ holds if and only if $\|A\|\|B\| \in \overline{W\left(A^{*} B\right)}$. 
Proof of Theorem 3.1. Let $e^{i \theta} T=H_{\theta}+i K_{\theta}$ be the Cartesian decomposition of $e^{i \theta} T$. We remark that

$$
K_{\theta}=H_{\theta-\pi / 2},
$$

because $e^{i(\theta-\pi / 2)} T=-i e^{i \theta} T=K_{\theta}-i H_{\theta}$. we have

(i) $\Rightarrow\left(\right.$ ii). Since $w(T)=\sup _{\theta \in \mathbb{R}}\left\|H_{\theta}\right\|=\sup _{\theta \in \mathbb{R}}\left\|K_{\theta}\right\|$ by (2.3) and (3.1),

$$
\|T\|=\left\|e^{i \theta} T\right\|=\left\|H_{\theta}+i K_{\theta}\right\| \leq\left\|H_{\theta}\right\|+\left\|K_{\theta}\right\| \leq w(T)+w(T)=\|T\|,
$$

proving (ii).

(ii) $\Rightarrow$ (i). For any $\theta \in \mathbb{R}$, (ii) ensures $\left\|H_{\theta}\right\|\left\|K_{\theta}\right\| \in \overline{W\left(H_{\theta}^{*}\left(i K_{\theta}\right)\right)}$ by Theorem C, i.e., $-i\left\|H_{\theta}\right\|\left\|K_{\theta}\right\| \in \overline{W\left(H_{\theta} K_{\theta}\right)}$. Since $-i\left\|H_{\theta}\right\|\left\|K_{\theta}\right\|$ is a purely imaginary number and $\operatorname{Im}\left(H_{\theta} K_{\theta}\right)=\operatorname{Im}\left(H_{0} K_{0}\right)$ for all $\theta \in \mathbb{R}$, we have

$$
\left\|H_{\theta}\right\|\left\|K_{\theta}\right\|=w\left(H_{\theta} K_{\theta}\right)=\left\|\operatorname{Im}\left(H_{\theta} K_{\theta}\right)\right\|=\left\|\operatorname{Im}\left(H_{0} K_{0}\right)\right\| .
$$

Thus for all $\theta \in \mathbb{R}$,

$$
\left\|H_{\theta}\right\|+\left\|K_{\theta}\right\|=\|T\|, \quad\left\|H_{\theta}\right\|\left\|K_{\theta}\right\|=\left\|\operatorname{Im}\left(H_{0} K_{0}\right)\right\|,
$$

that is,

$$
\begin{aligned}
\left\|H_{\theta}\right\| & =\frac{\|T\|+\sqrt{\|T\|^{2}-4\left\|\operatorname{Im}\left(H_{0} K_{0}\right)\right\|}}{2}, \\
\left\|K_{\theta}\right\| & =\frac{\|T\|-\sqrt{\|T\|^{2}-4\left\|\operatorname{Im}\left(H_{0} K_{0}\right)\right\|}}{2},
\end{aligned}
$$

or the other way round. We remark that these values do not depend on $\theta \in \mathbb{R}$. So the function $\left\|H_{\theta}\right\|$ of $\theta \in \mathbb{R}$ takes only two values by (3.1). By an easy calculation, we have

$$
H_{\theta}=H_{0} \cos \theta-K_{0} \sin \theta .
$$

Hence by the continuity of the operator norm, the function $\left\|H_{\theta}\right\|$ is continuous on $\theta \in \mathbb{R}$. Therefore it must be constant, i.e.,

$$
\left\|H_{\theta}\right\|=\left\|K_{\theta}\right\|=\frac{1}{2}\|T\| .
$$

Hence we have (i).

\section{References}

[1] A. Aluthge, Some generalized theorems on p-hyponormal operators, Integral Equations Operator Theory 24 (1996), 497-501.

[2] T. Ando, Aluthge transforms and the convex hull of the eigenvalues of a matrix, Linear Multilinear Algebra 52 (2004), 281-292.

[3] M. Barraa and M. Boumazgour, Inner derivations and norm equality, Proc. Amer. Math. Soc. 130 (2002), 471-476.

[4] T. Furuta, Invitation to Linear Operators, Taylor and Francis, London, 2001.

[5] K. E. Gustafson and D. K. M. Rao, Numerical Range, Springer, New York, 1997. 
[6] E. Heinz, Beiträge zur Störungstheorie der Spektralzerlegung, Math. Ann. 123 (1951), $415-438$.

[7] I. B. Jung, E. Ko and C. Pearcy, Aluthge transforms of operators, Integral Equations Operator Theory 37 (2000), 437-448.

[8] F. Kittaneh, A numerical radius inequality and an estimate for the numerical radius of the Frobenius companion matrix, Studia Math. 158 (2003), 11-17.

[9] P. Y. Wu, Numerical range of Aluthge transform of operator, Linear Algebra Appl. 357 (2002), 295-298.

[10] T. Yamazaki, An expression of spectral radius via Aluthge transformation, Proc. Amer. Math. Soc. 130 (2002), 1131-1137.

[11] - On numerical range of the Aluthge transformation, Linear Algebra Appl. 341 (2002), 111-117.

Department of Mathematics

Kanagawa University

Yokohama 221-8686, Japan

E-mail: yamazt26@kanagawa-u.ac.jp

Received April 7, 2006

Revised version September 25, 2006 